\title{
The Relationship Between Diabetes-Related Attitudes and Patients' Self-Reported Adherence
}

\author{
ROBERTM. ANDERSON, EdD; JAMES T. FITZGERALD, PhD; MARYS. OH, BS
}

This study involved 1202 patients

who were placed into low

adherence or high adherence

groups based on their answers to questionnaires. The attitudes of

each group were compared for a variety of adherence behaviors.

Patients who reported high levels of adherence tended to have attitudes more in accord with diabetes experts. Members of the high adherence group strongly supported the need for special training for health care professionals who treat diabetes, favored team care, accepted the importance of patient compliance, acknowledged the seriousness of non-insulin-dependent diabetes mellitus (NIDDM), and recognized the relationship between glucose control and complications. Differences in attitudes between high-and low adherence groups were more prevalent for difficult adherence areas, eg, diet and exercise, than for easy adherence areas, eg. carnying sweets or diabetic identification. An understanding of patients' attitudes can help diabetes educators and patients develop realistic and relevant self-care plans.
Diabetes is a lifelong chronic disease in which patients deliver over $95 \%$ of their own care. They must acquire the technical skills required for diabetes management in order to achieve a balance among medications, diet, exercise, and lifestyle. Patients also must learn how to adapt their daily lives to maximize their diabetes self-care while minimizing disruption of their lifestyles. Such a fit requires patients to develop clarity about their goals and priorities and to make a commitment to a self-care plan designed to achieve those goals.

The unique self-care character of diabetes makes patient education the crucial component of good diabetes care. Some of the major theories of health behavior and health education, such as the health belief model' and the theory of reasoned action. ${ }^{2}$ emphasize that attitudes and beliefs are major components of health behavior. Understanding the self-care behavior of patients with diabetes and responding to their needs with appropriate patient education requires some knowledge of their attitudes toward the disease and diabetes care. ${ }^{3}$

The theory of reasoned action' states that the best predictor of a patient's behavior is the patient's intention to behave in a certain way. For example, the theory suggests that the best predictor of whether a patient will carry out an intensive insulin regimen to obtain tight blood glucose control would be the patient's expressed intention of doing so. The theory of reasoned action further posits that a patient's intention to behave in a certain way involves two major determinants. The first determinant is the subjective norm, eg, whether people whom the patient views as important feel positively or negatively about the patient trying to achieve tight glucose control. The second determinant is the patient's own attitude toward the behavior, eg, how positively or negatively the patient feels about using an intensive insulin regimen to achieve tight blood glucose control. The patient's attitude is a summation of any and all of the patient's specific beliefs about the behavior. For example, Does the patient believe that an intensive insulin regimen will result in tight blood

From the University of Michigan Medical School, Michigan Diabetes Research and Training Center. Department of Postgraduate Medicine/Health Professions Education, Ann Arbor, Michigan.

This study was supported in part by a grant from the National Institute of Diabetes. Digestive, and Kidney Diseases (NIH). \#5P60DK20572, and the Diabetes Research and Education Foundation.

Correspondence to Robert M. Anderson. EdD. The University of Michigan Medical School. The Towsley Center, Room 11 16. Box 0201, Ann Arbor, MI 48109-0201.

Reprint requests to The Diabetes Educator, 500 North Michigan Avenue, Suite 1400, Chicago, IL 60611-3796. 
glucose control? Does the patient believe that tight blood glucose control will prevent complications?

The following study was conducted to examine the relationship between patients attitudes about diabetes and its treatment and their self-reported adherence to various aspects of the diabetes treatment regimen. We hope that the study will provide diabetes educators with insights about the relationship between patients' behaviors and attitudes that may be helpful in developing realistic, relevant. and acceptable diabetes self-care plans.

\section{Methods}

The Revised Diabetes Attitude Scale The study was conducted using a revised version of the Diabetes Attitude Scale (DAS) that measures the attitudes of both health care professionals and patients. The original DAS ${ }^{4.5}$ was developed to measure the attitudes of health care professionals by a national panel of 17 diabetes experts. This original version contained 50 statements reflecting a comprehensive range of issues concerning diabetes as a disease and the management of diabetes patient care. Each respondent indicated a degree of agreement, ranging from strongly agree through neutrality to strongly disagree, using a 5-point Likert-type scale. The DAS was evaluated psychometrically using the responses of 1071 health care professionals. Detailed results of the evaluation are reported elsewhere. ${ }^{4.5}$ The analysis identified eight factors representing attitudes toward: 1 ) the need for special training in the treatment of diabetes: 2) the importance of blood glucose control in minimizing the complications of diabetes: 3 ) the role of the patient in diabetes self-care and management; 4) the patients' commitment to controlling their disease: 5) the importance of a team approach to diabetes care; 6 ) the seriousness of non-insulin-dependent diabetes mellitus (NIDDM);7) the difficulties in treating diabetes: and 8) the efficacy of outpatient education.

While reviewing the responses to the DAS by health care professionals, it became clear that it would be useful to know how patients with diabetes viewed these same issues. The revision of the DAS to allow for its use in evaluating both the attitudes of patients and professionals (a Diabetes Attitude Scale to measure only patient attitudes would differ from the revised DAS) involved rewriting most of the 50 original statements to include less technical language while trying to retain the original meaning. Only nine items were left unchanged. Two random samples of health care professionals were selected: members of one group were sent the original DAS and the other group received the revised DAS. Responses from these two groups of health care professionals were analyzed and indicated that, despite the attempt to preserve the original meaning of each item, the revision process had changed the psychometric properties of the scale. ${ }^{6}$

Based on these results, it was determined that the revised DAS would have to be viewed as a new attitude measure. Accordingly, the psychometric properties were evaluated using responses from a sample of 1202 patients. ${ }^{7}$ The seven factors that were identified in this analysis were in good accord with the factors from the original DAS. These seven factors were: 1) the need for special training for health care professionals who treat diabetes; 2 ) the importance of patient compliance with medical advice; 3 ) the seriousness of
NIDDM; 4) the importance of blood glucose control in reducing diabetic complications; 5) the impact of diabetes on patients" lives; 6) the role of patient autonomy; and 7) the importance of a team approach to diabetes care. The differences in the factors between the two analyses reflected the differences in orientation of the two study populations. Patients focused on the impact of the disease upon individual lives, while health care professionals concerned themselves with difficulties in the treatment of the disease and the efficacy of patient education.

Study Participants In the present study, the responses of the 1202 patients to the revised DAS were used to explore the relationship between their attitudes and their self-reported adherence to various diabetes self-care recommendations. Surveys containing the revised DAS were mailed to two samples of patients with diabetes. The first sample consisted of 1054 patients who had attended the University of Michigan diabetes clinic. The survey was returned by $419 / 1054(40 \%)$ of the patients. To broaden the sample of patients, the survey also was sent to 1003 patients in the nine Michigan communities who were receiving a monthly diabetes newsletter. In this sample, the survey was returned by $823 / 1003$ (82\%) of the patients. Overall, 1242/2057 (60\%) surveys were returned. Forty surveys subsequently were eliminated from the analyses because the patients did not meet the age criterion (age $\geq 16$ years). Data from the final sample of 1202 patients were used for the present analysis.

The survey questionnaire asked patients to indicate whether they had insulin-dependent diabetes mellitus (IDDM) or non-insulin-dependent diabetes mellitus (NIDDM). Since previous surveys of patients indicated that a significant number do not know which type of diabetes they have, the questionnaire contained a brief explanation of each type of diabetes and a reminder that many patients with NIDDM also take insulin. However, despite these explanations. preliminary analysis of the data indicated that it was necessary to apply a formula developed by Davis et $\mathrm{al}^{8}$ to correctly classify the patients. The Davis formula utilizes age of onset. insulin use, and percent of ideal body weight to determine type of diabetes, and has been found to be $93 \%$ accurate when compared with the stimulated C-peptide test for classifying diabetes type.

Statistical Methods Demographic differences by type of diabetes were determined by chi-square analyses for nominal- and ordinal-scaled variables, and by analysis of variance for interval-scaled variables. Levels of patient self-report of adherence were determined for each of 10 self-care behaviors. A patient who reported performing the recommended self-care behavior "never," "rarely," or "sometimes" was placed in the low adherence group for that self-care behavior. A patient who reported performing the behavior "usually" or "always" was placed in the high adherence group.

Level of adherence to a particular self-care behavior was assigned only if patients indicated that the self-care behavior had been recommended to them by a health care professional. For the self-care behavior of testing urine for glucose levels, only patients who had not been told to perform blood glucose testing were assigned to an adherence group. For the self-care behavior of carrying sweets to treat low blood 
glucose levels, patients had to be using insulin to be assigned to a low- or high adherence group. Differences in the seven DAS subscales between high- and low adherence groups were determined by student $t$-tests. Due to the large number of tests, differences were considered significant at $P \leq .01$.

\section{Results}

Demographic Differences The characteristics of this population by type of diabetes and insulin use are provided in Table 1. Except for gender distribution, a statistically significant $(P \leq .01)$ difference was found for each demographic variable. Patients with IDDM were younger, had a longer duration of diabetes, had more formal education, and scored the highest on self-reported understanding of diabetes and on rating of overall health. Patients with NIDDM not using insulin reported the fewest complications, were the least likely to have attended a diabetes education program, and were least likely to report that their diabetes interfered with normal daily activities.

Self-Reported Adherence Patterns Listed in Table 2 are the self-reported adherence patterns for the 10 self-care behaviors. The majority of patients were in the high adherence group for each self-care behavior. The two self-care behaviors that had the largest number of patients reporting high adherence were taking insulin as instructed and taking diabetes pills as instructed. Low adherence to recommendations about exercise was reported by the largest number of patients.

Because so many patients were in the high adherence group regarding taking insulin $(97 \%$ ) or diabetes pills $(99 \%)$. these self-care behaviors were omitted from subsequent analyses. The self-care behavior of testing urine for sugar also was eliminated due to the small number of patients $(n=22)$ who were told to test their urine but not their blood glucose levels.

Adherence and Attitudes The seven diabetes attitude subscales that resulted from the factor analysis of the patient attitude responses ${ }^{7}$ were compared with adherence patterns. (All of the items in the revised DAS can be found in the report of this study.)

DAS Subscale I. Special Training This subscale reflects the attitude that health care professionals need special training to care for persons with diabetes. Sample item: "In general, I believe that to do a good job. diabetes educators should learn a lot about being teachers."

DAS Subscale 2, Patient Compliance This subscale reflects the attitude that patients should do what they are told to do by health care professionals. Several items on this subscale have a moralistic, blaming tone. Sample item: "In general, I believe that people who do not follow their recommended diabetes treatment do not really care about controlling their diabetes."

DAS Subscale 3, Seriousness of NIDDM This subscale reflects the attitude that NIDDM is a serious disease. Sample item: "In general, I believe that diabetes that can be controlled by just being on a diet is a pretty mild disease."

Table 1. Characteristics of Patients by Type of Diabetes

\begin{tabular}{|c|c|c|c|}
\hline & IDDM & $\begin{array}{l}\text { NIDDM } \\
\text { using } \\
\text { Insulin }\end{array}$ & $\begin{array}{c}\text { NIDDM } \\
\text { not using } \\
\text { Insulin }\end{array}$ \\
\hline Number studied & 410 & 445 & 324 \\
\hline$\%$ Female & 64 & 68 & 60 \\
\hline Mean age $(y)$ & 34.6 & 59.3 & 58.4 \\
\hline Mean duration $(y)$ & 17.9. & 13.5 & 9.8 \\
\hline $\begin{array}{l}\text { \% Family history of } \\
\text { diabetes }\end{array}$ & 56 & 65 & 61 \\
\hline $\begin{array}{l}\text { Mean number of } \\
\text { complications }\end{array}$ & 0.91 & 1.07 & 0.67 \\
\hline \% Sume college or more & 66 & 46 & 48 \\
\hline $\begin{array}{l}\text { \% Attended diabetes } \\
\text { education }\end{array}$ & 80 & 83 & 63 \\
\hline $\begin{array}{l}\text { Mean understanding } \\
\text { diabetes* }\end{array}$ & 4.15 & 3.58 & 3.32 \\
\hline $\begin{array}{l}\text { Mean diabetes prevents } \\
\text { activities } \dagger\end{array}$ & 2.01 & 1.86 & 1.37 \\
\hline Mean overall health $\ddagger$ & 3.39 & 2.99 & 3.11 \\
\hline $\begin{array}{l}* \text { Scoring: } 1=\text { poor to } 5=\text { excel } \\
\uparrow \text { Scoring: } 1=\text { never to } 5=\text { frec } \\
\ddagger \text { Scoring: } 1=\text { poor to } 5=\text { excel }\end{array}$ & & & \\
\hline
\end{tabular}

Table 2. Advised Self-Care Treatment of Diabetes and Self-Reported Adherence Patterns

\section{Advised Self-Care \\ Behaviors}

Taking insulin as instructed

Taking diabetes pills as instructed

Following a diabetic diet

Exercising

Testing blood for glucose

Testing urine for glucose $\ddagger$

Inspecting feet

Carrying diabetic identification

Carrying sweets (insulin users)

Recording test results

\begin{tabular}{|c|c|c|}
\hline $\mathbf{n}$ & $\begin{array}{c}\text { High- } \\
\text { Adherence } \\
\text { Group* } \\
\%\end{array}$ & $\begin{array}{c}\text { Low- } \\
\text { Adherence } \\
\text { Group } \dagger \\
\%\end{array}$ \\
\hline 838 & 97 & 3 \\
\hline 267 & 99 & 1 \\
\hline 1162 & 73 & 27 \\
\hline 1055 & 57 & 43 \\
\hline 1075 & 75 & 25 \\
\hline 22 & 73 & 27 \\
\hline 1045 & 78 & 22 \\
\hline 936 & 84 & 16 \\
\hline 778 & 84 & 16 \\
\hline 953 & 74 & 26 \\
\hline $\begin{array}{l}\text { or "usu } \\
\text { s," "ra }\end{array}$ & or "never" & \\
\hline
\end{tabular}

*High-adherence group: "always" or "usually"

tLow-adherence group: "sometimes," "rarely," or "never"

$\ddagger$ If not told to test blood for glucose

DAS Subscale 4, Control/Complications This subscale reflects the perception of a relationship between high blood glucose levels and the development of the complications of diabetes. Sample item: "In general, I believe that good blood sugar control will reduce the long-term complications of diabetes." 
Table 3. Mean Scores* for each Attitude Subscale for Patients in High-Adherence and Low-Adherence Groups

\begin{tabular}{|c|c|c|c|c|c|c|c|c|}
\hline $\begin{array}{l}\text { Self-Care } \\
\text { Behavior }\end{array}$ & $\begin{array}{l}\text { Adherence } \\
\text { Group }\end{array}$ & $\begin{array}{c}1 \\
\text { Special } \\
\text { Training }\end{array}$ & $\begin{array}{c}2 \\
\text { Patient } \\
\text { Compliance }\end{array}$ & $\begin{array}{c}3 \\
\text { Seriousness } \\
\text { of NIDDM }\end{array}$ & $\begin{array}{c}4 \\
\text { Control/ } \\
\text { Complications }\end{array}$ & $\begin{array}{c}5 \\
\text { Impact of } \\
\text { Diabetes }\end{array}$ & $\begin{array}{c}6 \\
\text { Patient } \\
\text { Autonomy }\end{array}$ & $\begin{array}{c}7 \\
\text { Team } \\
\text { Care }\end{array}$ \\
\hline \multirow{2}{*}{$\begin{array}{l}\text { Following a } \\
\text { diabetic diet }\end{array}$} & High & $4.30 \dagger$ & $3.64 \dagger$ & 3.47 & $4.28 \dagger$ & $4.08 \dagger$ & 3.58 & $4.19 \dagger$ \\
\hline & Low & 4.23 & 3.24 & 3.42 & 4.17 & 4.19 & 3.64 & 4.09 \\
\hline \multirow[t]{2}{*}{ Exercising } & High & 4.30 & $3.64 \dagger$ & 3.49 & 4.30 & $4.04 \dagger$ & 3.56 & 4.17 \\
\hline & Low & 4.27 & 3.40 & 3.47 & 4.24 & 4.20 & 3.66 & 4.18 \\
\hline \multirow[t]{2}{*}{ Testing blood } & High & $4.32 \dagger$ & $3.59 \dagger$ & $3.51 \dagger$ & $4.29 \dagger$ & 4.15 & 3.60 & $4.21 \dagger$ \\
\hline & Low & 4.20 & 3.32 & 3.34 & 4.17 & 4.10 & 3.69 & 4.05 \\
\hline \multirow[t]{2}{*}{ Inspecting feet } & High & 4.31 & $3.62 \dagger$ & 3.50 & 4.28 & 4.13 & 3.59 & $4.20 \dagger$ \\
\hline & Low & 4.24 & 3.30 & 3.40 & 4.24 & 4.11 & 3.67 & 4.11 \\
\hline \multirow{2}{*}{$\begin{array}{l}\text { Carrying diabetic } \\
\text { identification }\end{array}$} & High & 4.31 & $3.57 \dagger$ & 3.50 & 4.28 & 4.16 & 3.61 & 4.21 \\
\hline & Low & 4.24 & 3.37 & 3.39 & 4.20 & 4.15 & 3.68 & 4.16 \\
\hline \multirow[t]{2}{*}{ Carrying sweets } & High & 4.31 & $3.51 \dagger$ & 3.50 & 4.28 & 4.18 & 3.65 & 4.19 \\
\hline & Low & 4.23 & 3.26 & 3.39 & 4.26 & 4.21 & 3.78 & 4.08 \\
\hline \multirow[t]{2}{*}{ Recording tests } & High & $4.32 \dagger$ & $3.56 \dagger$ & 3.51 & $4.31 \dagger$ & 4.16 & $3.59 \dagger$ & $4.22 \dagger$ \\
\hline & Low & 4.20 & 3.31 & 3.40 & 4.18 & 4.14 & 3.47 & 4.06 \\
\hline
\end{tabular}

*Scoring: $1=$ strongly disagree to $5=$ strongly agree

$\nmid$ †ifference between high- and low-adherence groups is significant at $P \leq .01$

DAS Subscale 5, Impact of Diabetes This subscale reflects the attitude that diabetes has a significant negative impact on the patient's life. Sample item: "In general, I believe that diabetes affects almost every part of a diabetic person's life."

DAS Subscale 6, Patient Autonomy This subscale reflects the attitude that the patient should be the primary decision maker regarding the daily self-care of diabetes. Sample item: "In general. I believe that people with diabetes should choose their own goals for diabetes treatment."

DAS Subscale 7, Team Care This subscale reflects the attitude that nurses and dietitians are needed in the care of diabetes. Sample item: "In general, I believe that doctors should send people with diabetes to a nurse educator to help them learn about their diabetes."

Generally, patients in the high adherence group had more positive attitudes than patients in the low adherence group. There were three self-care behaviors (following a diet, testing blood glucose, recording results of blood or urine tests) in which the adherence groups differed on five of the seven diabetes attitude subscales (Table 3 ).

For the self-care behavior of following a diabetic diet, patients in the high- and low adherence groups differed on the attitudes concerning special training for health professionals, patient compliance, glucose control and complications, the negative impact of diabetes, and the team care approach. The patients in the high adherence group scored lower only for the attitude concerning the negative impact of diabetes. For the self-care behavior of testing blood glucose, patients in the high adherence group had higher scores for the attitudes concerning special training for health professionals, patient compliance, the seriousness of NIDDM, glucose control and complications, and the team care approach.
For the self-care behavior of recording blood or urine test results, patients in the high adherence group scored higher on the attitudes of special training for health professionals, patient compliance, glucose control and complications, patient autonomy, and the team care approach.

There was only one attitude subscale in which a difference was observed between the patients in the high- and low adherence groups for all seven self-care behaviors. Patients in the high adherence group for all of the self-care behaviors rated the patient compliance subscale higher than did their counterparts in the low adherence group.

\section{Discussion}

Following a Diet Seventy-three percent of the patients reported always or usually following their recommended diet. This figure probably represents some degree of bias consistent with self-reports of behaviors such as diet..,10 Patients in the group practicing high adherence to diet tended to have more positive attitudes in most areas. These patients also reported that diabetes had a less negative impact on their lives. Patients in the high adherence group expressed stronger agreement with statements about the importance of patient compliance. A number of these items, however, seem to have an inappropriate judgmental or blaming tone. For example, consider two items on the patient compliance subscale: "People who do not follow the recommended diabetes treatment don't really care about controlling their diabetes," and "Controlling their diabetes should be the most important thing in the lives of people with diabetes." Identifying a relationship between high adherence and a high degree of self-blame suggests that high levels of self-reported adherence to a particular aspect of the diabetic regimen (especially a difficult aspect such as diet or exercise) should not automatically be considered good. If patients report high adherence to a plan that they helped negotiate, one that is 
designed to help them reach their own diabetes care goals, such reports should be viewed in a positive light. However, these data indicate that patients are expressing agreement with judgmental and blaming statements about their own behavior or the behavior of other patients with diabetes. At the same time, the data also indicate that support for patient autonomy is relatively weak.

Two negative interpretations are possible for the relationship between high adherence and a high degree of selfblame. The first interpretation is that there may be some patients who deliberately misrepresent as good their actually poor adherence to a diet plan, since they view poor compliance as cheating or a failure. These patients may choose not to be open and honest in reporting their behavior to others or perhaps not even to themselves. Such a situation would be counterproductive. It is crucial for good diabetes education and for successful health care of any kind that an atmosphere of openness and trust exist between the patient and the health care advisors, and that a patient be honest with himself or herself.

A second interpretation is that there are some patients who are being compliant and adhere to diet plans without having a strong internalized commitment to or understanding of their diabetes care goals. These patients may be responding in a passive way to the perceived power and authority of health care professionals. The problem with this type of response is that diabetes requires a life-long commitment to thoughtful, intelligent self-care. It is unlikely that such a commitment can be sustained for long without a real internalization of the purposes and the value of good diabetes self-care.

However, previous attitude research ${ }^{7}$ has indicated that some patients prefer a traditional, hierarchical, authoritarian approach to diabetes care, while others prefer to take a more active approach of autonomy and self-direction. The diabetes educator's approach to promoting adherence to diet or any other aspect of the regimen should be related to the needs and expectations of individual patients.

Exercising Only $57 \%$ of patients reported high adherence to their recommended exercise regimen, attesting to the difficulty that patients have incorporating exercise into their daily lives. Patients who reported high adherence to exercise recommendations expressed stronger agreement on the patient compliance subscale and lower agreement on the subscale for negative impact of diabetes. Patients who feel successful in their efforts to take care of their diabetes by following recommendations regarding diet and exercise on a regular basis may be less likely to feel that the disease has a negative impact on their lives.

Blood Glucose Testing and Recording About $75 \%$ of the patients in this sample reported high adherence to the recommendations for testing their blood glucose levels and recording the results. The attitudes of patients in the high adherence group tended to be more positive than the attitudes of patients in the low adherence group, except for agreeing with the judgmental statements about patient compliance. High adherence patients expressed a stronger belief than low adherence patients in the relationship between the blood glucose levels and complications, the value of team care, the need for special training, and the seriousness of NIDDM. All of these attitudes are consistent with, and supportive of, regular use of blood glucose testing and recording of results.

Foot Inspection Because foot care involves a series of distinct behaviors, the survey focused on the most fundamental behavior, eg, regular inspection of the feet. Adherence to a recommendation to inspect the feet probably represents a moderate level of difficulty compared with adherence to following a diabetes diet, exercising, blood glucose testing (which we considered difficult), and carrying diabetic identification and sweets (which we considered easy). Not surprisingly, as one moves from behaviors that are difficult to behaviors that are easier, the size of the high adherence group increases and the difference in attitudes diminishes between the two groups. For example, the patients reporting high adherence to regular foot inspection differed from low adherence patients on only two attitude subscales, patient compliance and team care. This finding suggests that differences in attitudes are perhaps more relevant to adherence when difficult and challenging self-care behaviors are involved than when the behaviors are less difficult.

Carrying Diabetes Identification and Sweets These two behaviors may be two of the easiest recommendations for patients to follow. In these two areas, patients reporting high adherence differed from patients reporting low adherence only on the subscale of patient compliance. This finding may suggest that there are some patients for whom adherence is less a function of performing a particular behavior than it is a function of their inclination to accept or reject the recommendations of health care professionals.

Overall Adherence and Attitudes In general, patients who report high levels of adherence have more positive attitudes toward diabetes. They strongly support the need for special training for health care professionals to provide diabetes care and the importance of patient compliance, and they agree about the seriousness of NIDDM. They also express strong support for the relationship between glucose control and complications and importance of team care in diabetes. Furthermore, patients in the high adherence group for diet, exercise, and monitoring also reported higher levels of understanding diabetes and better overall health than patients in the low adherence group. Patients in both high- and low adherence groups strongly endorse the notion that diabetes has a negative impact on patients' lives and support the concept of patient autonomy, even if in moderation.

\section{Recommendations for Diabetes Educators}

This study further supports the principle that diabetes educators should assess and discuss the individual patient's attitudes and beliefs, especially in relationship to the more challenging and difficult aspects of the treatment regimen. Particular beliefs may constitute barriers or supports for helping patients make a commitment to good diabetes selfcare. Although some diabetes educators view increased adherence as the major purpose of diabetes patient education, we do not necessarily recommend this approach. We believe self-reported adherence can be a useful way of understanding what having and treating diabetes means to particu- 
lar patients. We view patient education as a process designed to enable patients to make informed choices about their diabetes self-care. Understanding the difficulties and challenges patients face in adhering to different aspects of the regimen can provide educators with useful information. This information can contribute to a greater understanding of a particular patient's beliefs about good diabetes self-care, which will focus and guide the patient's acquisition of appropriate skills and knowledge needed for the pursuit of relevant. realistic, and freely chosen goals for diabetes care.

\section{References}

1. Becker MH, Janz NK. The health belief model applied to understanding diabetes reginen compliance. Diabetes Educ 1985;11:\$1-47.

2. Ajzen $I$, Fishbein $M$. Understanding attitudes and predicting social behavior. Englewood Cliffs. NJ: Prentice Hall, 1980.

3. Anderson RM. Nowacek GW. Richards F. Influencing the personal meaning of diabetes: research and practice. Diabetes Educ 1988;14: 297-302
4. Anderson RM, Gressard C. Developing a measure of the attitudes of health care providers towards diabetes and its treatment. Diabetes 1987:36: 120A.

5. Anderson RM. Donnelly MB, Gressard C, Dedrick RF. The development of a diabetes attitude scale for health care professionals. Diabetes Care 1989:12:120-27.

6. Anderson RM, Donnelly MB. Words and meanings: a cautionary tale for diabetes educators. Diabetes Educ 1990:16:117-22.

7. Anderson RM, Donnelly MB. Dedrick RF. Measuring the attitudes of patients towards diabetes and its treatment. Patient Educ Couns 1990:16:231-45.

8. Davis WK, Hess GE, Hiss RG. Classification of diabetes types from clinical data. Diabetes 1988:37:260A.

9. Stunkard AJ, Waxman M. Accuracy of self-reports of food intake. J Am Diet Assoc 1981;79:547-51.

10. Owyer FT, Krall EA, Coleman KA. The problem of memory in nutritional epidemiology research. J Am Diet Assoc 1987;87:1509-12. 